\title{
4-Views Display System for Collaborative Tasks on Round Table
}

\author{
Mitsuru Okumura and Kunio Sakamoto \\ Department of Intelligence and Informatics, Konan University \\ 8-9-1 Okamoto, Higashinada, Kobe 658-8501, Japan
}

\begin{abstract}
This paper describes 4-views display system that can be viewed from any direction (i.e., the display has four viewing zones so as to perceive a screen view of the display at all directions around a table). The authors have ever researched information display systems involving 3D imaging. However, a conventional monitor display is viewed from one direction, that is, the display has narrow viewing angle and observers cannot view the screen from the opposite side. Hence we developed a tabletop display system for collaborative tasks cooperated by two users. This tabletop display can provide different images to two users surrounding the system utilizing the image splitting technologies for displaying a stereoscopic 3D image. The viewing zones of this display are generated at both sides in front of observers' eyes. But screens on the monitor cannot be viewed correctly by all users from any direction. Thus, conventional display systems enable users not to do collaborative tasks on the round table. To solve this problem, we developed new viewing systems.
\end{abstract}

Keywords: all around viewing, group work, grating film, table-top display.

\section{All-Around Viewing System}

To enable all-around viewing from four directions, a virtual screen is generated and floating above the top level of an actual display panel. Many techniques have been ever proposed in order to float images and locate pseudo images at different places from original positions. To simplify an optical layout, the authors utilize a grating sheet. The grating sheet provides a diffracted image which is arranged under or above original position. The grating sheet diffracts or scatters a light beam with a designed angle. This interesting phenomenon reminds us of method to shift image positions by a simple optical layout. Our developed a 4-views display system consists of four LCD panels and a square pyramidal optical screen. Fig. 1 shows a display unit and its layout constituted of four panels. Fig. 2 shows a square pyramid for generating four virtual screens above original positions of the actual display panels. An LCD display produces their image by having a liquid crystal layer that when a current runs through the pixel, it turns on that shade of color. The problem with the liquid crystal is that this color can only be accurately represented when viewed straight on. The further away from a perpendicular viewing angle, the color will tend to wash out. Thus, LCD displays have a limited viewing angle. As an observer watches a viewing screen on the panel with overlooking through the grating sheet, it loses contrast and becomes hard to read at out of the viewing angle as shown in Fig. 3(a). The snapshot of Fig. 3(a) is 
taken from upward diagonal direction. To correct visual clarity of an LCD's viewing screen, the LCD panels are covered with the grating sheet because a grating diffracts a ray into upwards and downwards. Although the snapshot of Fig. 3(b) is taken from upwards at same angle, it has more contrast and is easier to read at out of the viewing angle using the grating sheet.

A square pyramidal optical screen consists of four grating sheets on its surface so that the four virtual viewing screens are floating in the air. We shaped the viewing screen into a solid pyramid in order to enable all-around viewing from any directions. The grating sheet on the pyramid surface drifts a viewing screen in front of an observer's face. But an observer perceives not only a front view but also neighboring views. To solve this trouble, it is necessary to block lights of both neighboring views. Although louver film can also restrict a viewing angle, we utilized polarizers in consideration for easiness to obtain. The polarizer can switch whether a light goes through or not. If polarizers oriented at 90 degrees to each other, no light gets through. Meanwhile the polarizer passes a light wave when arranged for the same directions. As shown in Fig. 1 and Fig. 2, the LCD panel and the pyramid surface are covered by polarizers. For example, the horizontally oriented polarizers are attached on north and south display panels and east and west panels are covered with the vertical polarizers. North and south pyramid surfaces are also covered with the horizontal polarizers. East and west surfaces have the vertical polarizers too. The lights of both neighboring LCDs are blocked by the polarizers because polarizers on the LCD panel and the pyramid surface are oriented at 90 degrees to each other. An observer can perceive the only forward display through polarizers which have the same direction of polarization.
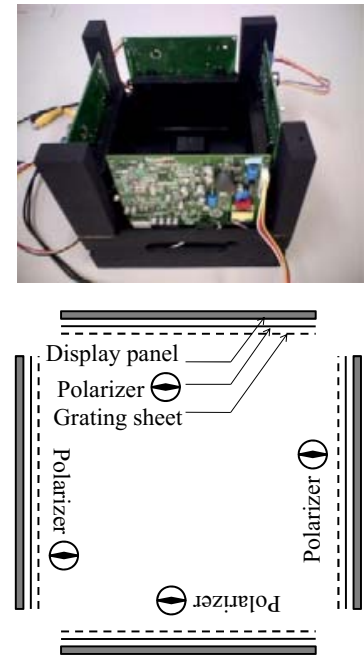

Fig. 1. Display unit
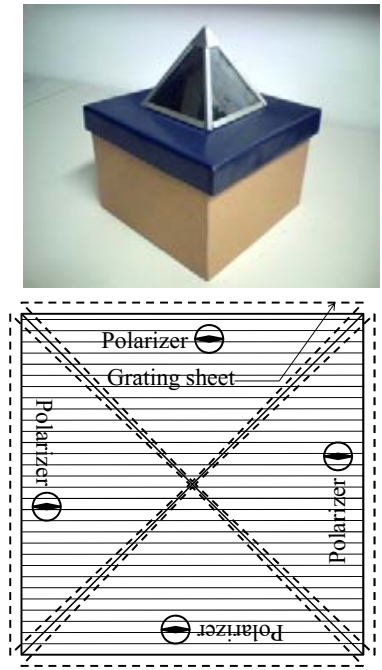

Fig. 2. Square pyramid

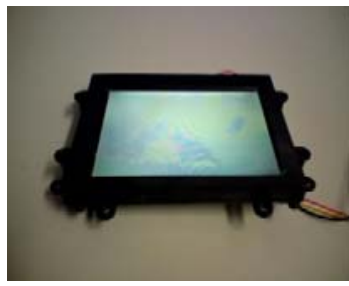

(a) no grating sheet

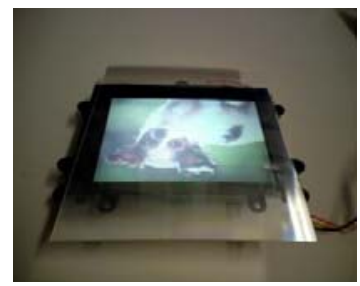

(b) grating attached

Fig. 3. Grating sheet

Acknowledgments. This research is partially supported by "Grant-in-Aid for Young Scientists(B)" \#20700112 and "Scientific Research (C) (General)" \#20500481 from Ministry of Education, Culture, Sports, Science and Technology Japan(MEXT) and also by a grant from the Hyogo Science and Technology Association. 